\title{
PRESENCE OF MULTIPLE TENDINOUS INSERTIONS OF PALMARIS LONGUS: A UNIQUE VARIATION OF A RETROGRESSIVE MUSCLE
}

\author{
Kumar Naveen ${ }^{1}$, Patil Jyothsna ${ }^{1}$, Swamy Ravindra S $^{1}$, Shetty Surekha D ${ }^{1}$, \\ Abhinitha $\mathbf{P}^{1}$, Rao Mohandas KG ${ }^{1}$, Nayak Satheesha $B^{1}$
}

\begin{abstract}
BACKGROUND: Palmaris longus, being the most variable muscle of the upper extremity is generally considered important morphologically rather than functionally. The palmar aponeurosis represents the distal part of the tendon of Palmaris longus.

CASE DETAILS: We report here a unique case of palmaris longus presenting variation distally as its tendon divides to gain multiple attachments with the fascia covering the abductor pollicis brevis, flexor retinaculum and the tendon of flexor carpi ulnaris. In addition, it also continues as palmar aponeurosis as its normal course.

CONCLUSION: Presence of this kind of variation might result in compression of the median and ulnar nerves and may aggravate the complications in case of inflammation of palmar aponeurosis thus mimicking the manifestations of Dupuytren's contracture.

KEYWORDS: Palmaris longus, palmar aponeurosis, Dupuytren's contracture.
\end{abstract}

DOI: http://dx.doi.org/10.4314/ejhs.v24i2.11

\section{INTRODUCTION}

Palmaris longus (PL), phylogenetically degenerated flexor muscle of metacarpophalangeal joint, takes origin from medial epicondyle of humerus as a common flexor origin, and converges distally as a long tendon which crosses the flexor retinaculum superficially. Tendon of the Palmaris longus crosses the retinaculum and fans out as a flat sheet which eventually continues as palmar aponeurosis. The median nerve at the wrist lies partly under cover of the tendon of PL (1).

Variations of PL muscle are not uncommon. It has been estimated that in about $11 \%$ of cases, they are found to be absent $(2,3)$. However, a study conducted by Mangala et al reported the agenesis of this muscle in $26 \%$ of individuals (4). Thus, it is often considered more important morphologically than functionally (5) as the palmar aponeurosis is considered to be the degenerated distal part of the PL which marks the probable evidence of retrogression of this muscle. PL is most popular in use as tendon grafts for the wrist without producing any functional deformities. However, variations in its distal attachment are often linked with median nerve and or ulnar nerve compressions. Therefore, variation involving PL cannot be overlooked clinically.

Prevalence of absence of PL tendon has been reported to be between $5.5 \%$ and $24 \%$ in Caucasian populations and between $4.6 \%$ and $26.6 \%$ in Asian population (6). Prevalence of its absence is reported to be higher in females than males, more common in left side than in right side $(2,3,7)$ and bilateral rather than in unilateral (3). Variant insertion of PL into thenar eminence (8), to retinaculum has been reported (9).

1Department of Anatomy, Melaka Manipal Medical College (Manipal campus), Manipal University, Manipal. Karnataka, India

Corresponding Author: Patil Jyothsna, Email: patiljyothsna@gmail.com 
Dupuytren's contracture is a progressive disorder characterized by localized thickening and contracture of palmar aponeurosis. Thus, the consequences rendered by the presence of anomalous multiple tendinous insertion of PL may be associated with the symptoms of Dupuytren's contracture.

\section{CASE REPORT}

During routine dissection for the medical undergraduate students, we observed a unilateral variation in the insertion pattern of the palmaris longus on the right upper limb of a male cadaver aged about 60 years. Proximally, the palmaris longus took its normal origin from the medial epicondyle of the humerus along with the common flexor tendons. The muscle belly formed the tendon distally and about $5 \mathrm{~cm}$ above the flexor retinaculum, the tendon bifurcated into two divisions-medial and lateral (Figure 1). The lateral division further subdivided into 2 slips. The lateral slip of the lateral division was attached to fascia covering the abductor pollicis brevis muscle and its medial slip continued further as the apex of the palmar aponeurosis. The broad medial division merged with the lateral border of the tendon of the flexor carpi ulnaris and flexor retinaculum.

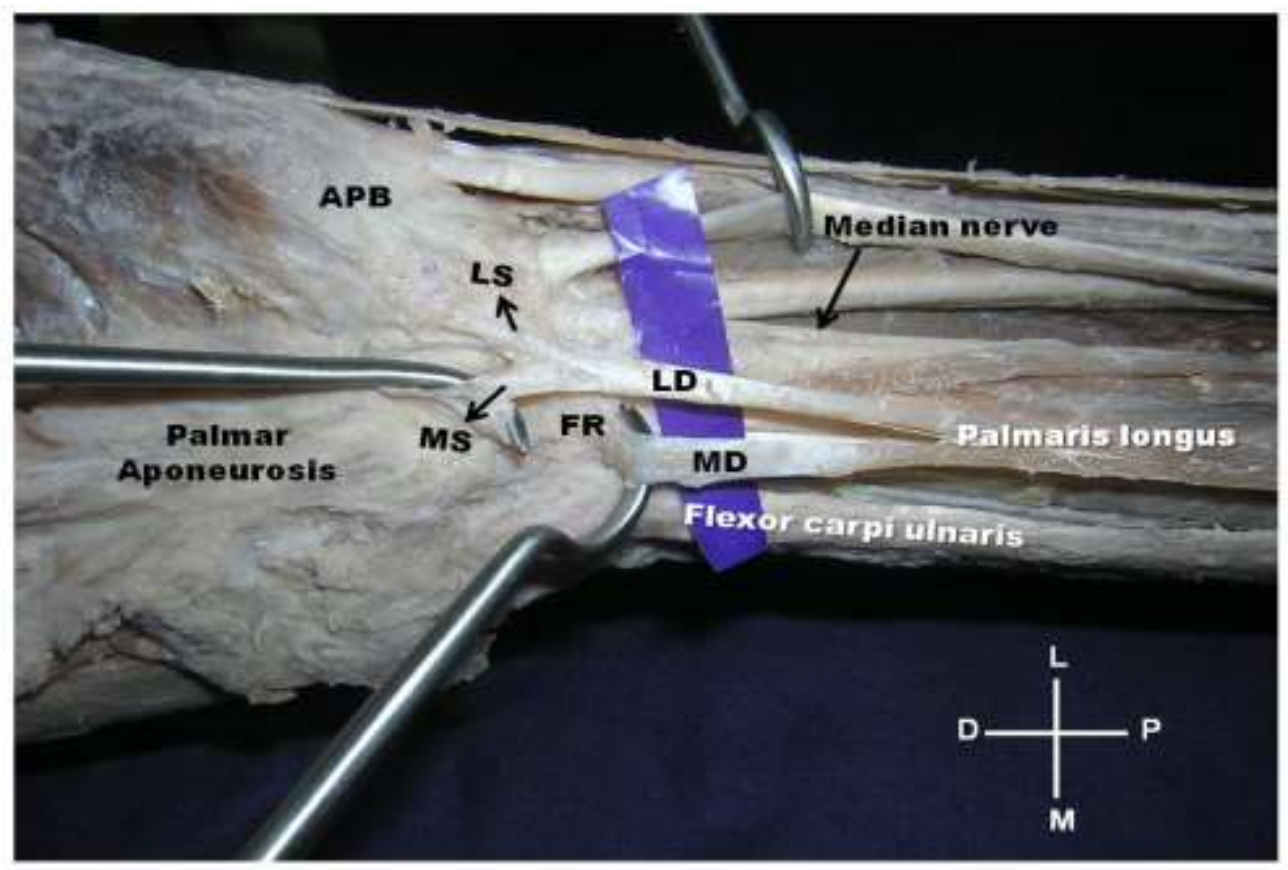

Figure 1: Tendon of palmaris longus dividing into medial $(M D)$ and lateral $(L D)$ divisions and splitting up of $L D$ into medial $(M S)$ and lateral $(L S)$ slips. APB: abductor pollicis longus. FR: flexor retinaculum, MN: median nerve. (L-lateral, $M$ - medial. D-dorsal, $P$ - proximal)

\section{DISCUSSION}

Palmaris longus is known to show variations. Reversed palmaris longus condition is one among its common polymorphic variation in which the muscle is fleshy distally and tendinous proximally $(7,10)$. Comprehensive summary of reports on reversed palmaris longus, associated with or without compressing the median nerve, by various authors has been reported by Valeria (10). Individual case reports by various authors documented the variant pattern of distal attachment of the muscle to the tendon of the flexor carpi ulnaris, abductor pollicis brevis muscle, and/or fascia of the forearm, fascia or muscles of hypothenar eminence, any of the long flexor tendons or at the site of metacarpophalangeal joints etc $(2,3)$. However, report on unique variation with multiple division of tendon of the palmaris longus is not available in the literature survey. Variations at its site of insertion are clinically important as it is closely 
related to median and ulnar nerves. Sufficient number of reports has been published about the compression of median nerve by palmaris longus (7, 11-15) as well as ulnar nerve compression (16). On the other hand, hypertrophic palmaris longus can results in pseudo mass of the forearm (17).

Dupuytren's contracture commonly begins at the root of the ring finger and draws it into palm. Later, this condition generally extends into the little finger. Pull on fibrous flexor sheath results in flexion at interphalangeal joints (18). This contracture results in flexion deformity of the fingers and loss of hand function with the acute flexion of proximal and middle phalanges as the palmar aponeurosis is attached to them (19). The variations as we observed here with multiple abnormal attachments of the palmaris longus may aggravate these symptoms with the possible involvement of the thumb. Abnormal attachment to the fascia covering the abductor pollicis brevis muscle may affect the abduction of the thumb. In addition, the tendon of the palmaris longus is commonly used for grafting purposes by the hand surgeons. Variant insertion pattern often misleads the surgeons in such approaches. Therefore, while performing PL graft procedures, surgeons should be aware of this kind of variation in order to avoid accidental damage to the median nerve.

To conclude, multiple divisions of tendon of PL at its site of insertion into neighboring structures cannot be overlooked in the scenario of the inflammation of the palmar aponeurosis mimicking the symptoms of Dupuytren's contracture and compression of the median or ulnar nerves.

\section{REFERENCES}

1. Standring S; Grays anatomy: The Anatomical basis of clinical practice, Elsevier churchil living stone (39 ${ }^{\text {th }}$ edition) $2005 ; 876-77$.

2. Bergman RA, Thompson SA, Afifi AK. Catalog of human variation. Urban \& Schwarzenberg: Baltimore. 1984.

3. Tountas CP, Bergman RA. Anatomic variations of the upper extremity. Churchill Livingstone: New York. 1983.

4. Mangala M P, Latha V P, Nayak SR, Sampath M, Rajanigandha V, Krishnamurthy A, Kumar A. The Palmaris longus muscle: its anatomic variations and functional morphology.
Romanian Journal of Morphology and Embryology, 2008;49(2):215-217.

5. Chaurasia B D, Human Anatomy: Regional and applied dissection and clinical, Vol 2 (Lower Limb, Abdomen \& Pelvis)-4th edition. CBS Publishers, 2004;94-95.

6. Kose O, Adanır O, Cirpar M, Kurklu M, Komurcu M. The prevalence of absence of the palmaris longus: a study in Turkish population. Arch Orthop Trauma Surg, 2009; 129(5): 609-11.

7. Depuydt KH, Schuurman AH, Kon M. Reversed Palmaris longus muscle causing effort-related median nerve compression. $J$ Hand Surg 1998; 23B(1):117-119.

8. Vasanth K, Bincy M G. An unusual Palmaris longus tendon: Variation in the insertion and orientation at the level of wrist joint. IJAV, 2009; 2:138-139.

9. Natsis K, Didagelos M, Manoli SM, Vlasis K, Papathanasiou E, Sofidis G, Xerantzidou X. Fleshy Palmaris longus muscle- A cadaveric finding and its clinical significance: Case report. Hippokratia, 2012; 16 (4): 378-380.

10. Valeria P Sassoli F Reversed Palmaris Longus muscle and median nerve relationships: Case reports and literature review. Braz. J. Morphol. Sci, 2007;24 (2):88-91.

11. Giunta R, Brunner U, Wilhelm K. Bilateral reverser Musculus Palmaris longus - seltene Ursache eines peripheren N.-medianuskompressionssyndroms. Unfallchirurg, 1993; 96: 538-540.

12. Guler MM, Celiköz B. Anomalous Palmaris longus muscle causing carpal tunnel-like syndrome. Arch Orthop Trauma Surg, 1998; 117: 296-297.

13. Meyer FN, Pflaum BC. Median nerve compression at the wrist caused by a reversed Palmaris longus muscle. J Hand Surg [Am], 1987;12: 369-371.

14. Schlafly B, Lister G. Median nerve compression secondary to bifid reversed Palmaris longus. J Hand Surg [Am], 1987; 12: 371-373.

15. Seyhan T. Madian nerve compression at the wrist caused by reversed 3-headed Palmaris longus muscle: Case report and review of the literature. Am J Orthop, 2005; 34: 544-546.

16. Regan PJ, Roberts JO, Bailey BN. Ulnar nerve compression caused by a reversed Palmaris 
longus muscle. J Hand Surg [Br], 1988; 13: 406-407.

17. Polesuk B. S, Helms C. A. Hypertrophied palmaris longus muscle, a pseudomass of the forearm: MR appearance - case report and review of the literature. Radiology, 1998; 207(2):361-362.
18. Richard S. Clinical anatomy (Snell) by regions $\left(8^{\text {th }}\right.$ ed.). Wolter Kluwer- Lippincott Williams \& Wilkins, 2008.

19. Datta AK. Essentials of Human Anatomy (Superior \& inferior extremities) $3^{\text {rd }}$ edition. Current books international Kolkata. 2004. 